\title{
Hamiltonian approach to the torsional anomalies and its dimensional ladder
}

\author{
Ze-Min Huang, ${ }^{1, \text { 冈 }}$ Bo Han, ${ }^{2,1}$ and Michael Stone ${ }^{1}$ \\ ${ }^{1}$ Department of Physics, University of Illinois at Urbana-Champaign, \\ 1110 West Green Street, Urbana, Illinois 61801, USA \\ ${ }^{2}$ Theory of Condensed Matter Group, Cavendish Laboratory, University of Cambridge, \\ J. J. Thomson Avenue, Cambridge CB3 OHE, United Kingdom
}

(Dated: June 4, 2020)

\begin{abstract}
Torsion can cause various anomalies in various dimensions, including the $(3+1)$-dimensional $[(3+1) D]$ Nieh-Yan anomaly, the $(2+1)$ D Hughes-Leigh-Fradkin (HLF) parity anomaly, and the $(3+1) \mathrm{D},(1+1) \mathrm{D}$ chiral energy-momentum anomaly. We study these anomalies from the Hamiltonian approach. We derive the $(1+1) \mathrm{D}$ chiral energy-momentum anomaly from the single-body Hamiltonian. We then show how other torsional anomalies can be related to the $(1+1) \mathrm{D}$ chiral energy-momentum anomaly in a straightforward way. Finally, the Nieh-Yan anomaly and the $(3+1) \mathrm{D}$ chiral energy-momentum anomaly are obtained from the parity anomaly and the HLF effective action, respectively. Hence, we have constructed the dimensional ladder for the torsional anomalies from the single-body Hamiltonian picture.
\end{abstract}

\section{INTRODUCTION}

Many topological phases of matter can be understood from quantum anomalies [1 [5]. A classic example is the integer quantum Hall effect, where the Hall current is captured by the $(2+1)$-dimensional parity anomaly in the bulk and thus the $(1+1)$-dimensional gauge anomaly at the edges [6]. Both the electromagnetic anomalies and the gravitational anomalies have been extensively used to characterize various topological phases of matter [5, 7[15]. Especially, the dimensional ladder for these anomalies has been known for a long time [16]. Namely, the chiral anomaly, the parity anomaly and the gauge anomaly in different dimensions relate to each other closely, which has been used to classify topological phases of matter in different dimensions [3, 17.

Torsion can cause quantum anomalies as well, but they are less studied compared to the electromagnetic anomalies and the gravitational anomalies. However, torsion is ubiquitous in condensed matter. The torsion tensor can be used as a probe for thermal transport [18 20, and also to describe dislocations 21 24. In addition, torsion can also emerge from a order parameters of Fermi superfluid or topological superconductors [25]27]. Hence, torsional effects in topological phases of matter are attracting more and more attention and they can lead to various novel phenomena, including the anomalous thermal Hall effect in Weyl semimetals 28, 29, the chiral torsional magnetic effect [30] and other viscoelastic responses [22, 24, 31, 37]. Due to the close relation between topological phases of matter and quantum anomalies, it is thus natural to ask what torsional anomalies we have and whether they form a dimensional ladder.

Various torsional anomalies have been obtained in the context of high-energy physics [23, 24, 38, 43, including the Nieh-Yan chiral anomaly and the chi-

\footnotetext{
* zeminh2@illinois.edu
}

ral energy-momentum anomaly (or the chiral diffeomorphism anomaly) in four dimensions 24, 38 43, the Hughes-Leigh-Fradkin (HLF) parity anomaly [44] in three dimensions [22, 23] and the chiral energymomentum anomaly in two dimensions [23]. In the same spirits as Ref. [45], these anomalies can be derived from the Atiyah-Patodi-Singer index theorem or the chiral anomaly 24. However, the torsional anomalies listed here are all divergent and thus depend on the ultraviolet physics. Hence, an alternative approach based on the Hamiltonian is highly desired, which enables us to appreciate the physics behind these divergences because the Hamiltonian approach is more familiar to condensed matter physicists.

In this paper, we derive various torsional anomalies based on the Hamiltonian approach. First, the $(1+1)$ dimensional chiral energy-momentum anomaly is derived from the single-body Hamiltonian. Then, all other torsional anomalies can be straightforwardly obtained from this anomaly, including the $(2+1)$-dimensional HLF effective action, the $(3+1)$-dimensional Nieh-Yan anomaly and the $(3+1)$-dimensional chiral energy-momentum anomaly. Especially, we show that the quadratic cutoff in the $(1+1)$-dimensional chiral energy-momentum anomaly is the energy density, so the cutoff measures the depth of the vacuum. We also reveal the close relation between the $(3+1)$-dimensional torsional anomalies and the $(2+1)$-dimensional parity-odd effective action. Namely, assuming translational invariance along one direction, the corresponding momentum is a good quantum number, so it acts like a mass term. The response currents from the $(2+1)$-dimensional effective action are then used to derive the corresponding $(3+1)$-dimensional anomaly currents at a given momentum. The corresponding $(3+1)$-dimensional anomaly current can thus be obtained by summing over all the momentum. Finally, motivated by the recently predicted thermal NiehYan anomaly [29, 46, 47], we conjecture that there are thermal corrections to the torsional anomalies in the dimensional ladder obtained above, where the dimensionful 
coefficients are replaced by the temperature. Although we have focused on the 4-3-2 dimensional ladder, our results can be easily generalized to $(2 n+2)-(2 n+1)-$ $(2 n)$ dimensional ladder, which is discussed in the two appendixes.

The rest of this paper is organized as follow. In Sec. III we give a brief summary of the torsional anomalies. In Sec. [II] we construct the first part of the dimensional ladder shown in Fig. 1. i.e., the $(1+1)$-dimensional chiral energy-momentum anomaly, the $(2+1)$-dimensional HLF effective action, and the $(3+1)$-dimensional chiral energy-momentum anomaly. In Sec. IV] we construct the second part of the dimensional ladder for the chiral current, i.e., $(1+1)$-dimensional chiral $U(1)$ anomaly, $(2+1)$-dimensional parity anomaly, and $(3+1)$-dimensional Nieh-Yan anomaly. We summarize the main results of this paper in Sec. $\mathrm{V}$

\section{TORSIONAL ANOMALIES}

It is known that torsion can induce various anomalies, including the Nieh-Yan anomaly in four dimensions [38, 39, 41, the chiral energy-momentum anomaly in two and four dimensions [23, 24] and the HLF parity anomaly in three dimensions. Interestingly, all these anomalies contain a divergent coefficient and thus depend on the ultraviolet cutoff. To be more concrete, the Nieh-Yan anomaly is (for the derivation using Fujikawa's method, please refer to Appendix A

$\frac{1}{\sqrt{|g|}} \partial_{\mu} \sqrt{|g|} j^{5 \mu}=\frac{\Lambda^{2}}{32 \pi^{2}} \frac{\epsilon^{\mu \nu \rho \sigma}}{\sqrt{|g|}}\left(T^{a}{ }_{\mu \nu} T_{\rho \sigma}^{b} \eta_{a b}-2 e_{\mu}^{* a} e_{\nu}^{\star b} \Omega_{a b \rho \sigma}\right)$,

where $\Lambda$ is the cutoff, $e_{\mu}^{\star a}$ is the vielbein and $g_{\mu \nu}$ is the metric with $g \equiv \operatorname{det} g_{\mu \nu} . \quad a, \mu=0,1,2,3$ are the coordinates indices: $a$ is called the Lorentz index and it labels the locally flat coordinates with basis $\left\{e_{a}\right\}$, while $\mu$ is called the Einstein index and it is used for the basis $\left\{\partial_{\mu}\right\} . j^{5 \mu}$ is the axial current. $T^{a}{ }_{\mu \nu}$ is the torsion tensor, i.e., $T^{a}=\frac{1}{2} T^{a}{ }_{\mu \nu} d x^{\mu} \wedge d x^{\nu}$ and $T^{a}=d e^{\star a}+\omega^{a}{ }_{b} \wedge e^{\star b}$, where $\omega^{a}{ }_{b}=\omega^{a}{ }_{b \mu} d x^{\mu}$ is the spin connection. $\Omega_{a b \mu \nu}$ is the curvature tensor, i.e., $\Omega_{a b}=\frac{1}{2} \Omega_{a b \mu \nu} d x^{\mu} \wedge d x^{\nu}$ and $\Omega_{a b}=d \omega_{a b}+\omega_{a}^{c} \wedge \omega_{c b}$.

As for the massless Dirac fermions in $(1+1)$ dimensional space-time, we have (for details, refer to Appendix B

$$
\begin{aligned}
& \nabla_{\mu} \tau_{(c) a}^{5 \mu}+T_{\mu \rho}^{\rho} \tau_{(c) a}^{5 \mu}-e_{a}^{\mu} T_{\mu \nu}^{d} \tau_{(c) d}^{5 \nu} \\
= & \left(\frac{\Lambda^{2}}{4 \pi}\right) \eta_{a b} \frac{\epsilon^{\mu \nu}}{\sqrt{|g|}} T^{b}{ }_{\mu \nu}+\ldots,
\end{aligned}
$$

where ... means that we have kept only the leading divergent terms. $\tau_{(c) a}^{\mu}$ and $\tau_{(c) a}^{5 \mu}$ are the canonical energymomentum tensor and the chiral canonical energymomentum tensor, respectively. $\eta_{a b}=\operatorname{diag}(1,-1)$ is the metric. Terms in the first line of Eq. (2) can be derived from the chiral covariant translational symmetry. That is, the right-handed Weyl fermions transform as $\psi_{R} \rightarrow \xi^{\mu} D_{\mu} \psi_{R}$, and $\psi_{L} \rightarrow-\xi^{\mu} D_{\mu} \psi_{L}$ for lefthanded Weyl fermions (for details, refer to Appendix B), where $D_{\mu}$ is the Lorentz covariant derivative and it acts on the Lorentz indices. So these terms appear even at the classical limit. Compared with the U(1) case, fermions coupled to the electromagnetic field have a classical equation of motion for the energy-momentum tensor as $\nabla_{\mu} \tau^{\mu \nu}=F^{\nu \mu} j_{\mu}$, where $F^{\nu \mu}$ is the field strength tensor of the electromagnetism. In both cases, we treat the electromagnetic field or the torsion field as the external background field. The terms in the second line are the anomalies from the quantum fluctuations. In the same spirit as the Callan-Harvey mechanism [48], this term is expected to originate from the current inflow in the bulk, which will be explored in the next section. This chiral energy-momentum anomaly appears in four dimensions as well. Especially, for the massless Dirac fermions,

$$
\begin{aligned}
& \nabla_{\mu} \tau_{(c) a}^{5 \mu}+T_{\mu \rho}^{\rho} \tau_{(c) a}^{5 \mu}-e_{a}^{\nu}\left(T_{\mu \nu}^{d} \tau_{(c) d}^{5 \mu}-\Omega_{c d, \mu \nu} S^{\mu c d}\right) \\
= & \frac{\Lambda^{2}}{16 \pi^{2}} \eta_{a b} \frac{\epsilon^{\mu \nu \rho \sigma}}{\sqrt{|g|}} F_{\mu \nu} T_{\rho \sigma}^{b}+\ldots,
\end{aligned}
$$

where ... means that only the most divergent terms are kept. Detailed calculations are relegated to Appendix $B$ Finally, for the $(2+1)$-dimensional massive Dirac fermions, the parity-odd effective action induced by torsion is

$$
S_{\mathrm{HLF}}=\frac{m^{2} \operatorname{sgn}(m)}{8 \pi} \int d^{3} x \epsilon^{\mu \nu \rho} e_{\mu}^{a} T_{\nu \rho}^{b} \eta_{a b},
$$

where $m$ is the mass of Dirac fermions. Note that by direct perturbative calculations [22], this effective action is divergent, and it is proportional to the cutoff $\Lambda$. However, for reasons that will be discussed in the next section, the HLF action here is written in terms of the mass instead of the cutoff.

Clearly, the coefficients of the anomaly terms in Eq. (2), (3), and Eq. (4) are all dimensionful and divergent. One may be curious whether we can understand these anomalies in a consistent way, or they are simply an artificial illness. For Dirac fermions in curved spacetime without torsion, it is known that the chiral anomaly, the parity anomaly, and the gauge anomaly consist of a dimensional ladder [45. Namely, the $(2 n+1)$ dimensional parity anomaly can be obtained from the $(2 n+2)$-dimensional chiral anomaly by using the AtiyahPatodi-Singer index theorem. Due to the Callan-Harvey mechanism, the current inflow captured by the $(2 n+1)$ dimensional parity odd effective action (parity anomaly) leads to the (2n)-dimensional gauge anomaly at the edges. By employing the same idea, the torsional anomalies can be derived as well [24]. However, it is also tempting to derive these torsional anomalies from the singlebody Hamiltonian, which emphasizes the physical mechanism behind these torsional anomalies. Finally, it is worth mentioning that in Ref. [27], the cut-off in the 


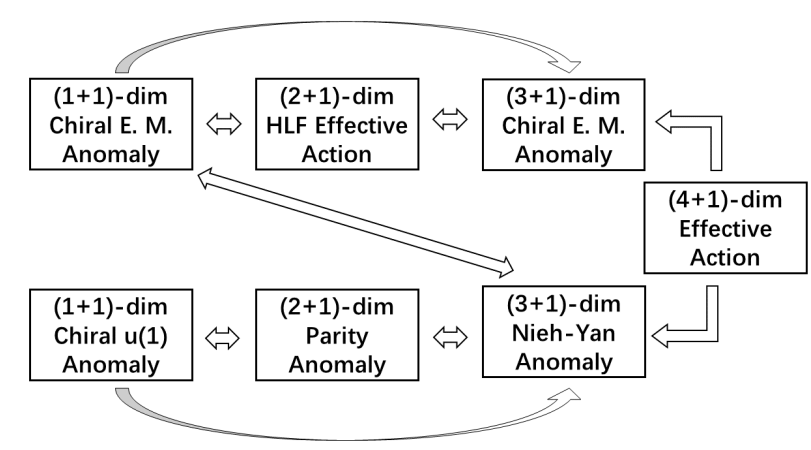

Figure 1. Dimensional ladder for the torsional anomalies. "Chiral E. M. Anomaly" stands for the chiral energymomentum anomaly. "HLF effective action" stands for the Hughes-Leigh-Fradkin effective action obtained in Ref. [22]. The boxes in the first line are for the anomalies related to the energy-momentum tensor (the corresponding derivation based on the Hamiltonian approach is given in Sec. III), while boxes in the bottom are for the anomalies in the chiral U(1) current (the corresponding derivation is presented in Sec. IV].

Nieh-Yan anomaly is argued to be proportional to the Fermi momentum in the ${ }^{3} \mathrm{He}-A$ superfluid.

\section{ANOMALIES OF THE CHIRAL ENERGY-MOMENTUM TENSOR}

In this section, we shall study the anomalies encoded in the chiral energy-momentum tensor based on the Hamiltonian approach (boxes in the first row in Fig. 1). First of all, we shall derive the $(1+1)$-dimensional chiral energy-momentum anomaly from the single-body Hamiltonian. Then, both the $(2+1)$-dimensional HLF effective action and the $(3+1)$-dimensional chiral energymomentum anomaly can easily be obtained from the $(1+1)$-dimensional chiral energy-momentum anomaly. Finally, we further show how to derive the $(3+1)$ dimensional chiral energy-momentum anomaly from the $(2+1)$-dimensional HLF effective action, which completes the first row of the dimensional ladders for the torsional anomalies shown in Fig. 1.

\section{A. (1+1)-dimensional chiral energy-momentum anomaly from the single-body Hamiltonian}

The Hamiltonian for the $(1+1)$-dimensional Dirac fermions is

$$
H=\left(-i \partial_{z}\right) \sigma^{3},
$$

where $\sigma^{3}$ is the Pauli matrix, i.e., $\sigma^{3}=\operatorname{diag}(1,-1)$ and the chirality of the fermions is labeled by $s= \pm 1$. Then, we turn on the vielbein. For simplicity, we assume that $e_{\mu}^{\star a}=\delta_{\mu}^{a}+\delta_{3}^{a} \delta_{\mu}^{z} \Phi(t)$ with $\partial_{t} \Phi \ll 1$ and $\Phi \ll 1$. The corresponding torsional electric field is given as $T^{3}{ }_{E}=$ $\partial_{t} e_{z}^{\star 3}-\partial_{z} e_{t}^{\star 3}=\partial_{t} \Phi$. Because $e_{a}^{\mu}$ is defined from $e_{\mu}^{\star a}$ by requiring $e_{\mu}^{* a} e_{b}^{\mu}=\delta_{b}^{a}, e_{a}^{\mu} \simeq \delta_{a}^{\mu}-\delta_{z}^{\mu} \delta_{a}^{3} \Phi$. The coupling between the Pauli matrices and the vielbein is $\sigma^{a} \rightarrow \sigma^{a} e_{a}^{\mu}$, so the Hamiltonian becomes

$$
H=[1-\Phi(t)]\left(-i \partial_{z}\right) \sigma^{3} .
$$

Notice that $\partial_{t} \Phi(t) \ll 1$, so one can obtain the dispersion relation, i.e., $\mathcal{E}_{s}=s(1-\Phi) p_{z}$. In addition, the effective Fermi velocity is now $v_{F}=(1-\Phi)$, so $v_{F}$ can be tuned by changing the vielbein adiabatically. Hence, if we impose a cutoff on the energy, i.e., $-\Lambda<\mathcal{E}_{s}<\Lambda$, we can squeeze particles and thus momentum out of the system by tuning $\Phi$, which leads to the energy-momentum anomaly for $s$-fermions. To be more concrete, let us calculate the momentum density for $s$-fermions, i.e.,

$$
\begin{aligned}
\tau_{(c) 3}^{s t} & =\int \frac{d p_{z}}{2 \pi} n_{F}\left[\mathcal{E}_{s}\left(p_{z}\right)\right] p_{z} \\
& =\frac{1}{(1-\Phi)^{2}} \int_{-\Lambda s}^{+\Lambda s} \frac{d \mathcal{E}_{s}}{2 \pi} \frac{1}{\exp \left(\beta \mathcal{E}_{s}\right)+1} \mathcal{E}_{s} \\
& \stackrel{T \equiv 0}{=}-\frac{s}{4 \pi(1-\Phi)^{2}} \Lambda^{2},
\end{aligned}
$$

where $s= \pm 1$ stands for the chirality and $\mathcal{E}_{s}=s(1-\Phi) p_{z}$ is the dispersion relation. $\Lambda$ is the energy cutoff, i.e., $-\Lambda<(1-\Phi) p_{z}<\Lambda$, and it measures the depth of the vacuum. $n_{F}\left(\mathcal{E}_{s}\right)=\left[\exp \left(\beta \mathcal{E}_{s}\right)+1\right]^{-1}$ is the Fermi-Dirac distribution, with $\beta=\frac{1}{T}$ being the inverse of the temperature $T$. Equation (6) tells us that the averaged momentum density of the occupied electrons receives corrections from the vielbein. The energy cutoff is fixed at $\Lambda$, so the changes in $\Phi$ will change the Fermi velocity and thus the averaged momentum [23]. As a consequence, there is an anomaly in the momentum current for $s$-fermions.

From Eq. (6), the time derivative of the chiral momentum density at the small $\Phi$ approximation is

$$
\partial_{t} \tau_{(c) 3}^{5 t}=-\frac{\Lambda^{2}}{\pi} \partial_{t} \Phi
$$

or in a covariant form,

$$
\nabla_{\mu} \tau_{(c) a}^{5 \mu}=\frac{\Lambda^{2}}{2 \pi} \eta_{a b} \frac{\epsilon^{\mu \nu}}{\sqrt{|g|}} T^{b}{ }_{\mu \nu}+\ldots
$$

where ... stands for other terms appearing at the classical level. This is similar to the result in Eq. (2) up to a multiplier of 2 , but we can always rescale the cutoff to match them. The physical mechanism of this anomaly can be straightforwardly appreciated as follow. Let us consider the right-handed fermions $(s=+1)$ for concreteness. Since the chemical potential is set to zero, all the negative-energy states are occupied. The only energy scale in the system is the cutoff, so by dimensional analysis, the averaged momentum must be proportional to the cutoff square, i.e., $\tau_{(c) 3}^{s t} \propto \Lambda^{2}$. In the presence of vielbeins, i.e., $e_{z}^{* 3}=1+\Phi$, the cutoff for the momentum 
becomes $(1+\Phi) \Lambda$. Hence, the change in momentum density is $\Delta \tau_{(c) 3}^{s t} \propto \Lambda^{2}-(1+\Phi)^{2} \Lambda^{2}=-2 \Phi \Lambda^{2}$, which implies that $\frac{\Delta \tau_{(c) 3}^{s t}}{\Delta t} \propto-\Lambda^{2} \partial_{t} \Phi$, and this leads to the chiral energymomentum anomaly.

\section{B. Torsional anomalies from the $(1+1)$-dimensional chiral energy-momentum anomaly}

After obtaining the $(1+1)$-dimensional chiral energymomentum anomaly, one can straightforwardly obtain other torsional anomalies, including the $(2+1)$ dimensional HLF effective action, the $(3+1)$-dimensional chiral energy-momentum anomaly, and the Nieh-Yan anomaly.

As for the HLF effective action, this can be done by employing the Callan-Harvey mechanism [23, 24, 48]. Consider the $(2+1)$-dimensional Chern insulators with chiral zero modes at the edges. Then, the $(1+1)$-dimensional chiral energy-momentum anomaly derived in last section implies the existence of the energy-momentum anomalies for the chiral edge modes. This energy-momentum anomaly can only come from the current inflow in the bulk, so the corresponding energy-momentum current in the bulk must be

$$
\tau_{(c) a}^{\mu}=\frac{m^{2} \operatorname{sgn}(m)}{4 \pi} \frac{\epsilon^{\mu \nu \rho}}{\sqrt{|g|}} \eta_{a b} T_{\nu \rho}^{b},
$$

where $m$ is the gap of the $(2+1)$-dimensional Chern insulators and it acts as the cut-off for the edges states, so $\Lambda$ in Eq. (2) is replaced by $m$. In addition, $\operatorname{sgn}(m)$ is from the chirality of the edges modes. Note that we are considering the continuum model in the bulk rather than the tight-binding model, so the prefactor is $\operatorname{sgn}(m)$. Alternatively, if the tight-binding model is considered, then $\operatorname{sgn}(m)$ should be replaced by $1-\operatorname{sgn}(m)$. This difference comes from different regularization schemes.

From Eq. (8), one can easily write down the corresponding effective action, i.e.,

$$
S_{\mathrm{HLF}}=\frac{m^{2} \operatorname{sgn}(m)}{8 \pi} \int d^{3} x \epsilon^{\mu \nu \rho} e_{\mu}^{* a} T_{\nu \rho}^{b},
$$

where the canonical energy-momentum tensor is defined as $\tau_{(c) a}^{\mu}=\left.\frac{-1}{\sqrt{|g|}} \frac{\delta S}{\delta e_{\mu}^{* a}}\right|_{\omega_{a b \mu}}$ with the spin connection $\omega_{a b \mu}$ kept fixed. Hence, we have recovered the $(2+1)$-dimensional HLF effective action from the $(1+1)$-dimensional chiral energy-momentum anomaly.

Interestingly, the $(3+1)$-dimensional Nieh-Yan anomaly can be derived from the $(1+1)$-dimensional chiral energy-momentum anomaly as well. Consider Weyl fermions under a specific configuration of vielbeins, i.e., $e_{\mu}^{* a}=\delta_{\mu}^{a}+w_{\mu}^{a}$ and $w_{\mu}^{a}=\frac{1}{2} \delta_{3}^{a} \tilde{T}_{B}^{3}(0,-y, x, 0), \tilde{T}_{B}^{3}>0$, which means that the torsional magnetic fields are applied along the $z$ direction. For simplicity, the spin connection is set to zero. Then, the effective Hamiltonian for Weyl fermions with $s$-chirality is

$$
\begin{aligned}
H_{s}= & s\left[p_{z} \sigma^{3}+\left(\hat{p}_{x}+\frac{1}{2} \tilde{T}_{B}^{3} y p_{z}\right) \sigma^{1}\right. \\
& \left.+\left(\hat{p}_{y}-\frac{1}{2} \tilde{T}_{B}^{3} x p_{z}\right) \sigma^{2}\right],
\end{aligned}
$$

where $p_{z}$ is a good quantum number and $s= \pm 1$ denotes the chirality. This Hamiltonian looks like Weyl fermions under magnetic fields $\tilde{T}_{B}^{3}$ with charge $p_{z}$. The dispersion relation can be straightforwardly derived, i.e.,

$$
\mathcal{E}_{s}=\left\{\begin{array}{cc}
s\left|p_{z}\right| & n=0 \\
\pm \sqrt{p_{z}^{2}+2\left|n \tilde{T}_{B}^{3} p_{z}\right|} & |n| \geq 1
\end{array} .\right.
$$

Compared to the magnetic case, the lowest torsional Landau levels are $s\left|p_{z}\right|$ rather than $s p_{z}$, which is because the charge of the torsional magnetic fields is $p_{z}$ and it contributes an extra minus sign when $p_{z}<0$. Notice that only the lowest torsional Landau levels can distinguish Weyl fermions with different chiralities. Thus, the $|n|>1$ torsional Landau levels contribute equally to the $s$-Weyl fermion current. That is, as far as the chiral anomaly is concerned, only the lowest torsional Landau levels matter. Consequently, the system is effectively reduced from $(3+1)$-dimensional to $(1+1)$-dimensional and the corresponding effective $(1+1)$-dimensional Lagrangian is

$$
\mathcal{L}=\frac{1}{2}\left(\bar{\psi}^{\prime} \Gamma^{\mu} i \partial_{\mu} \psi^{\prime}-\bar{\psi}^{\prime} i \overleftarrow{\partial}_{\mu} \Gamma^{\mu} \psi^{\prime}\right)
$$

where $\Gamma^{\mu}$ is the $(1+1)$-dimensional gamma matrix, $\psi^{\prime}\left(p_{z}\right)=\left(\begin{array}{c}\psi_{R} \\ \psi_{L}\end{array}\right)$ for $p_{z}>0$, and $\psi^{\prime}\left(p_{z}\right)=\left(\begin{array}{c}\psi_{L} \\ \psi_{R}\end{array}\right)$ for $p_{z}<0$. The $(3+1)$-dimensional chiral current can be defined in terms of the $(1+1)$-dimensional Dirac fermions, i.e.,

$$
j^{5 \mu}=\left(\bar{\psi}^{\prime} \Gamma^{\mu} \Gamma^{5} p_{z} \psi^{\prime}\right)\left(\frac{\tilde{T}_{B}^{3}}{2 \pi}\right),
$$

where $\tilde{T}_{B}^{3}$ is the torsional magnetic field, $\frac{1}{2 \pi}\left(\left|p_{z}\right| \tilde{T}_{B}^{3}\right)$ is from the torsional Landau level degeneracy, and the chirality of $\psi^{\prime}$ is twisted by $\operatorname{sgn}\left(p_{z}\right)$. Especially, $\left(\bar{\psi}^{\prime} \Gamma^{\mu} \Gamma^{5} p_{z} \psi^{\prime}\right)$ is the $(1+1)$-dimensional chiral energy-momentum density $\tau_{(c) z}^{5(1+1) \mu}$. Hence, $\nabla_{\mu} j^{5 \mu}=$ $\left(\nabla_{\mu} \tau_{(c) z}^{5(1+1) \mu}\right) \tilde{T}_{B}^{3}$, where $\nabla_{\mu} \tau_{(c) a}^{5(1+1) \mu}$ is the $(1+1)$ dimensional chiral energy-momentum anomaly. Consequently, the Nieh-Yan anomaly in Eq. (1) can be derived from the $(1+1)$-dimensional chiral energy-momentum anomaly.

As for the $(3+1)$-dimensional chiral energymomentum anomaly in Eq. 3, it involves a term mixing the torsion tensor and the electromagnetic tensor. This anomaly can also be obtained from the $(1+1)$-dimensional chiral energy-momentum anomaly. Namely, under external magnetic fields, the $(3+1)$ dimensional Dirac fermions are effectively reduced to the 
$(1+1)$-dimensional ones consisting of the lowest Landau levels. The $(3+1)$-dimensional chiral energy-momentum anomaly can be written as $\nabla_{\mu} \tau_{(c) a}^{5(3+1) \mu} \propto \nabla_{\mu} \tau_{(c) a}^{5(1+1) \mu} B$, which coincides with the anomaly in Eq. (3). So far, we have shown that most known torsional anomalies can be understood from the $(1+1)$-dimensional chiral energy-momentum anomaly. It is natural to ask the relation between the $(2+1)$-dimensional HLF effective action and the $(3+1)$-dimensional torsional anomalies.

\section{C. (3+1)-dimensional chiral energy-momentum anomaly from the $(2+1)$-dimensional HLF effective action}

In this section, we shall reveal the relation between the HLF effective action and the $(3+1)$-dimensional chiral energy-momentum anomaly based on the single-body Hamiltonian.

The Hamiltonian for the $(3+1)$-dimensional righthanded Weyl fermions under torsion is

$$
H=H_{\perp}\left(\hat{p}_{x}, \hat{p}_{y}, e_{\perp \mu}^{* a}\right)+p_{z} \sigma^{3},
$$

where the background curvature is assumed to be zero, so we can set the spin connection to zero. $H_{\perp}\left(\hat{p}_{x}, \hat{p}_{y}, e_{\perp \mu}^{* a}\right)$ is the Hamiltonian for the $(2+1)$-dimensional massless Dirac fermions with vielbeins and $e_{\perp \mu}^{* a}=e_{\perp \mu}^{* a}(x, y)$ is the vielbein in the space perpendicular to $z$. In addition, $p_{z}$ is a good quantum number. Note that $p_{z} \sigma^{3}$ looks like a mass term for the $(2+1)$-dimensional Dirac fermions. So from the HLF effective action, the momentum density at a given momentum $p_{z}$ is

$$
\rho_{(c) a}^{(+) t}\left(p_{z}\right)=\frac{p_{z}^{2} \operatorname{sgn}\left(p_{z}\right)}{4 \pi} \eta_{a b} T_{B}^{b}
$$

where the superscript $(+)$ denotes the chirality, the mass term $m^{2} \operatorname{sgn}(m)$ is replaced by $p_{z}^{2} \operatorname{sgn}\left(p_{z}\right)$ and $T^{a}{ }_{B}=$ $-\left(\partial_{x} e_{y}^{\star a}-\partial_{y} e_{x}^{\star a}\right)$ is the torsional magnetic fields. Now let us further turn on the electric fields, i.e., $p_{z} \rightarrow p_{z}-A_{z}(t)$, where $\partial_{t} A_{z} \ll 1$. Thus, the momentum density at given momentum $p_{z}$ becomes

$$
\rho_{(c) a}^{(+) t}\left(p_{z}-A_{z}\right)=\frac{\left(p_{z}-A_{z}\right)^{2} \operatorname{sgn}\left(p_{z}-A_{z}\right)}{4 \pi} \eta_{a b} T_{B}^{b},
$$

which implies that the momentum density of the righthanded Weyl fermions can be tuned by varying $A_{z}$ adiabatically. Hence, the momentum for the right-handed Weyl fermions can be pumped up from the Dirac sea by changing $A_{z}$. Notice that the effective masses for the right-handed and left-handed Weyl fermions always have opposite signs. So the increasing of the momentum density of the right-handed Weyl fermions is accompanied by the decreasing of the momentum density of left-handed ones. Consequently, there is an anomaly in the $(3+1)$ dimensional chiral energy-momentum tensor, but not the energy-momentum tensor.
Now let us calculate the total momentum density by integrating over $p_{z}$. The total momentum density for right-handed Weyl fermions is given as

$$
\begin{aligned}
\tau_{(c) a}^{(+) t} & =\int_{-\Lambda}^{+\Lambda} \frac{d p_{z}}{2 \pi} \rho_{(c) a}^{t} \\
& =-\frac{\Lambda^{2}}{4 \pi^{2}} \eta_{a b} A_{z} T_{B}^{b}
\end{aligned}
$$

This implies that

$$
\partial_{t} \tau_{(c) a}^{5 t}=-\frac{\Lambda^{2}}{2 \pi^{2}} \eta_{a b} E_{z} T_{B}^{a}+\ldots
$$

and its covariant form is the $(3+1)$-dimensional chiral energy-momentum anomaly given in Eq. (3). Hence, we have completed the first part of the dimensional ladder shown in Fig. 1 .

\section{ANOMALIES OF THE CHIRAL CURRENT}

In the last section, we completed the dimensional ladder for the chiral energy-momentum anomaly. In this section, we shall show that there is an analog ladder for the anomalies encoded in the chiral current as well, which is shown in the second row in Fig. 1. Namely, the Nieh-Yan anomaly can be understood from the $(2+1)$-dimensional parity anomaly, and the $(2+1)$ dimensional parity anomaly can be obtained from the $(1+1)$-dimensional chiral anomaly.

The relation between the $(2+1)$-dimensional parity anomaly and the $(1+1)$-dimensional chiral anomaly is well known in the context of the integer quantum Hall effect, which is used to understand the bulk-edge correspondence. For completeness, we shall briefly review it here. The covariant form of the Hall current in $(2+1)$ dimensions is

$$
j^{\mu}=\frac{\operatorname{sgn}(m)}{8 \pi} \epsilon^{\mu \rho \sigma} F_{\rho \sigma},
$$

where $m$ is the mass of the $(2+1)$-dimensional Dirac fermions and $\operatorname{sgn}(m)$ is from the chirality of the edge modes. In addition, the spatial components of the current above can be recast in a more inspiring form, i.e., $\boldsymbol{j}=\frac{\operatorname{sgn}(m)}{4 \pi} \hat{z} \times \boldsymbol{E}$. If we consider the tight-binding model in the bulk instead, then the Hall current becomes $\boldsymbol{j}=$ $\frac{1}{2 \pi}\left[\frac{1-\operatorname{sgn}(m)}{2}\right] \hat{z} \times \boldsymbol{E}$, and $\frac{1}{2}[1-\operatorname{sgn}(m)]$ is the Chern number in the bulk. So for the topologically non-trivial phase, $\frac{1-\operatorname{sgn}(m)}{2}=1$, we have obtained the quantized Hall conductance, i.e., $\sigma_{H}=\frac{1}{2 \pi}$. From the Hall current in Eq. (19), one can write down the parity odd effective action (the parity anomaly), i.e.,

$$
S=\frac{\operatorname{sgn}(m)}{16 \pi} \int d^{3} x \epsilon^{\mu \rho \sigma} A_{\mu} F_{\rho \sigma} .
$$

In addition, Eq. 19 also tells us that there is current flowing toward the edges, which leads to the current 
nonconservation at the edges or the $(1+1)$-dim gauge anomaly.

Now let us turn to the Nieh-Yan anomaly and its relation to the parity anomaly. The Hamiltonian for the right-handed Weyl fermions is

$$
H=H_{\perp}\left(\hat{p}_{x}, \hat{p}_{y} ; e_{\perp \mu}^{* a}\right)+p_{z} \sigma^{z},
$$

where $p_{z}$ is a good quantum number and $H_{\perp}$ is the Hamiltonian for the $(2+1)$-dimensional Dirac fermions under external vielbeins, i.e., $e_{\perp a}^{\mu}=\delta_{a}^{\mu}-w_{\perp a}^{\mu}(x, y)$. For concreteness, we further assume that $H_{\perp}$ is given as

$$
H_{\perp}\left(\hat{p}_{x}, \hat{p}_{y} ; e_{\perp \mu}^{* a}\right)=\left(\hat{p}_{x}-w_{1}^{z} p_{z}\right) \sigma^{x}+\left(\hat{p}_{y}-w_{2}^{z} p_{z}\right) \sigma^{z} .
$$

Hence, Eq. 21) looks like $(2+1)$-dimensional massive Dirac fermions (the mass term is $p_{z} \sigma^{3}$ ) under external electromagnetic field $w_{i}^{z}$ with charge $p_{z}$. From the parity anomaly, one can straightforwardly read off the Hall current density at given momentum $p_{z}$, i.e.,

$$
\rho^{(+)}\left(p_{z}\right)=\frac{1}{4 \pi} p_{z} \operatorname{sgn}\left(p_{z}\right) T^{3}{ }_{B},
$$

where $T^{3}{ }_{B}$ is the torsional magnetic field, i.e., $T^{3}{ }_{B}=$ $\partial_{x} w_{y}^{3}-\partial_{y} w_{x}^{3}$. Now we further turn on the torsional electric fields, i.e., $T_{E}^{3}=\partial_{t} e_{z}^{* 3}-\partial_{z} e_{t}^{* 3}$. For simplicity, we set $e_{t}^{* 3}=0$ and $e_{z}^{* 3}=1+\Phi(t)$ with $\Phi$ being a slowly varying field. Hence, the Hall density becomes

$$
\rho^{(+)}\left(p_{z}\right)=\frac{1}{4 \pi} p_{z} \operatorname{sgn}\left[(1-\Phi) p_{z}\right] T_{B}^{3},
$$

where $e_{z}^{* 3}$ modifies only the mass term, and not the charge of the effective electromagnetic fields $w_{i}^{z}$. The total current density can be derived by summing over the momentum,

$$
\begin{aligned}
j^{(+) 0} & =\int \frac{d p_{z}}{2 \pi} \rho^{(+)}\left(p_{z}\right) \\
& =\frac{1}{8 \pi^{2}(1-\Phi)^{2}} \int_{-\Lambda}^{\Lambda} d \mathcal{E} \mathcal{E} \operatorname{sgn} \mathcal{E} T^{3}{ }_{B} \\
& \simeq \frac{1}{4 \pi^{2}} \Lambda^{2} \Phi T^{3}{ }_{B},
\end{aligned}
$$

where $\mathcal{E} \equiv(1-\Phi) p_{z}$ is the energy and we have imposed a cutoff $\Lambda$ upon the energy. By recasting the result above to a covariant form, one can obtain the Nieh-Yan anomaly in Eq. (1). This completes the second part of the dimensional ladder shown in Fig. 1 .

It is interesting to notice that the $(3+1)$-dimensional chiral energy-momentum anomaly involves the mixing term between the torsion and the electromagnetic tensor, while the Nieh-Yan anomaly involves only the torsion tensor. Furthermore, if we regard the $(3+1)$-dimensional Weyl fermions as the boundary chiral modes of some $(4+1)$-dimensional topological phases of matter, then from the Callan-Harvey mechanism, one can obtain the corresponding $(4+1)$-dimensional effective action [24]

$$
S_{(4+1)}=\frac{m^{2} \operatorname{sgn}(m)}{64 \pi^{2}} \int d^{5} x \epsilon^{\mu \nu \rho \sigma \delta} A_{\mu} T_{\nu \rho}^{a} T_{\sigma \delta}^{b} \eta_{a b},
$$

whose response charge current and energy-momentum current are the bulk origin of the boundary Nieh-Yan anomaly and chiral energy-momentum anomaly, respectively.

\section{SUMMARY AND DISCUSSION}

The dimensional ladder for torsional anomalies is constructed based on the single-body Hamiltonian. We have shown how to obtain the $(2+1)$-dimensional HLF effective action, the $(3+1)$-dimensional Nieh-Yan anomaly and chiral energy-momentum anomaly from the $(1+1)$ dimensional chiral energy-momentum anomaly. In addition, we have also clarified the relation between the $(2+1)$-dimensional HLF effective action and the $(3+1)$-dimensional chiral energy-momentum anomaly, the $(2+1)$-dimensional parity anomaly and the $(3+1)$ dimensional Nieh-Yan anomaly. Our work has provided a complete physical picture for various torsional anomalies in various dimensions.

Recently, it was pointed out in Ref. [29, 46, 47] that, at finite temperature, there is an extra thermal term in the Nieh-Yan anomaly as well, where the cut-off is replaced by the temperature. Hence, based on the dimensional ladder obtained here, it is natural to conjecture that all the torsional anomalies in Fig. 1 that are cutoff dependence receive thermal corrections as well. That is, the dimensionful parameter is replaced by the temperature. Specifically, for the $(1+1)$-dimensional chiral energy-momentum anomaly calculated in Eq. (6), if we use the finite-temperature Fermi-Dirac distribution function instead of the zero-temperature one, then there is an extra thermal term proportional to the temperature square. If this conjecture is true, then the $(2+1)$ dimensional thermal Hall effect can be straightforwardly understood from the $(2+1)$-dimensional thermal HLF effective action in the bulk, or $(1+1)$-dimensional energymomentum anomaly at the edges. In addition, the recently observed negative magneto-thermoelectric resistance reported in Ref. [14 can be understood based on this conjecture as well. Namely, if there is an extra thermal term in the $(3+1)$-dimensional chiral energymomentum anomaly equation in Eq. (3), i.e., $\nabla_{\mu} \tau_{a}^{5 \mu} \propto$ $T^{2} \frac{\epsilon^{\mu \nu \rho \sigma}}{\sqrt{|g|}} \eta_{a b} F_{\mu \nu} T_{\rho \sigma}^{b}+\ldots$, then by considering the inter valley scatterings, in the steady states, the chiral chemical potential is proportional to $\epsilon^{\mu \nu \rho \sigma} \eta_{a b} F_{\mu \nu} T_{\rho \sigma}^{a}$, or $T(\boldsymbol{B} \cdot \nabla T)$ for $a=0$. Together with the chiral magnetic effect, one can thus obtain the negative magnetothermoelectric resistance. However, here, we shall restrict ourselves to the zero-temperature limit and leave the conjecture for future study. 


\section{ACKNOWLEDGMENTS}

The authors wish to thank B. Bradlyn for insightful discussions. Z.-M.-H. and M.-S. were not directly supported by any funding agencies, but this work would not be possible without resources provided by the Department of Physics at the University of Illinois at UrbanaChampaign. B. H. was supported by ERC Starting Grant No. 678795 TopInSy.

\section{Appendix A: Fujikawa's approach to the Nieh-Yan anomaly}

In this appendix, we shall provide a detailed derivation of the torsion induced chiral anomaly. For convenience, we shall focus on the Euclidean space-time. The convention of the Wick rotation is

$$
t=-i \tau, \gamma_{E}^{0}=\gamma^{0}, \gamma_{E}^{i}=-i \gamma^{i}
$$

where $\tau$ is the Euclidean time. The subscript $E$ stands for the Euclidean spacetime, which will be neglected without causing any confusion, because in this appendix we will focus on the Euclidean space-time.

The Jacobian $J[\theta]$ of the chiral transformation $\psi \rightarrow$ $e^{i \theta \gamma_{5}} \psi$ is known to be 49

$$
\ln J[\theta]=-2 i \theta \operatorname{Tr} \gamma_{5}
$$

where $\operatorname{Tr}$ is the trace of both the internal indices and the spacetime coordinates. $\operatorname{Tr} \gamma_{5}$ is obviously divergent, so we introduce the following regulator,

$$
\exp \left(\frac{\not \mathcal{D}}{\Lambda^{2}}\right)
$$

where

$$
\not{D}=e_{a}^{\mu} \gamma^{a}\left(\partial_{\mu}+\frac{1}{2} \omega_{a b \mu} \sigma^{a b}+\frac{1}{2} T_{\mu \rho}^{\rho}\right)
$$

and

$$
\overleftarrow{\not{D}}=\left(\partial_{\mu}-\frac{1}{2} \omega_{a b \mu} \sigma^{a b}+\frac{1}{2} T_{\mu \rho}^{\rho}\right) e_{a}^{\mu} \gamma^{a}
$$

are skew Hermitian. Note that $\not D$ is neither Hermitian nor skew Hermitian, so we use $\mathcal{D}$ rather than $D$ and the extra term $\frac{1}{2} T_{\mu \rho}^{\rho}$ in $\not{D}$ and $\overleftarrow{\mathcal{D}}$ is canceled in the action.

Then, for $\not{D} \not \mathcal{D}$, there is

$$
\begin{aligned}
& (\not{D})^{2} \\
= & \delta^{a b} \mathcal{D}_{a} \mathcal{D}_{b}+\sigma^{a b}\left(-T^{d}{ }_{a b} \mathcal{D}_{d}+\frac{1}{2} \Omega_{c d, a b} \sigma^{c d}\right) \\
& +\sigma^{a b}\left(\partial_{a} T^{\rho}{ }_{b \rho}-\partial_{b} T^{\rho}{ }_{a \rho}\right)
\end{aligned}
$$

where we have used

$$
\left[D_{a}, D_{b}\right] \psi=\left(-e_{a}^{\mu} e_{b}^{\nu} T_{\mu \nu}^{d} D_{d}+\frac{1}{2} e_{a}^{\mu} e_{b}^{\nu} \Omega_{\mu \nu}\right) \psi
$$

and thus

$$
\begin{aligned}
& {\left[\mathcal{D}_{a}, \mathcal{D}_{b}\right] \psi } \\
= & {\left[-T_{a b}^{d} D_{d}+\frac{1}{2} \Omega_{c d, a b} \sigma^{c d}\right] \psi . } \\
& +\left(\partial_{a} T_{b \rho}^{\rho}-\partial_{b} T^{\rho}{ }_{a \rho}\right) \psi .
\end{aligned}
$$

Because we are most interested in the torsion, for simplicity, let us set the curvature $\Omega_{a b}=\frac{1}{2} \Omega_{a b \mu \nu} d x^{\mu} \wedge d x^{\nu}$ to zero. Hence, we can choose such a gauge that the spin connection is zero. In addition, in the presence of electromagnetic fields,

$$
(\not \mathcal{D})^{2} \simeq \delta^{a b} \mathcal{D}_{a} \mathcal{D}_{b}+\sigma^{a b}\left(-T^{d}{ }_{a b} D_{d}+i F_{a b}\right)
$$

where $\simeq$ is because that we are most interested in the most divergent terms, $\left(\partial_{a} T_{b \rho}^{\rho}-\partial_{b} T_{a \rho}^{\rho}\right)$ is neglected. Notice that in the absence of curvature,

$$
\left\{\mathcal{D}_{a}, \mathcal{D}_{b}\right\}=\partial_{b}\left(i A_{a}+\frac{1}{2} T_{a \rho}^{\rho}\right)+(a \leftrightarrow b)+\ldots
$$

so we can choose such a gauge fixing condition that $\partial_{a} A_{b}+\partial_{b} A_{a}=0$. In addition, we shall neglect the terms from $T_{\mu \rho}^{\rho}$ hereafter, which is reasonable because we are interested in only the most divergent terms.

Then, 


$$
\begin{aligned}
& -2 i \operatorname{Tr}\left(\gamma^{5} e^{\Lambda^{-2} \not \boldsymbol{D}}\right) \\
= & -2 i \sum_{n} \frac{1}{n !} \int d^{d} x \int \frac{d^{d} k}{(2 \pi)^{d}} e^{-\Lambda^{-2} k^{2}} \operatorname{tr}\left(\gamma^{5} \sigma^{a_{1} b_{1}} \sigma^{a_{2} b_{2}} \ldots \sigma^{a_{n} b_{n}}\right) \prod_{i=1}^{n}\left(-i \Lambda^{-2} T_{a_{i} b_{i}} k_{d}+i \Lambda^{-2} F_{a_{i} b_{i}}\right)^{n} \\
\rightarrow & -2 i \sum_{n} \frac{1}{n !} \int d^{d} x \int \frac{d^{d} k}{(2 \pi)^{d}} e^{-\Lambda^{-2} k^{2}}\left(-2 \Lambda^{-2} T^{d} k_{d}+2 \Lambda^{-2} F\right)^{n} \\
= & -2 i \sum_{n} \sum_{p=0,2,4 \ldots} C_{n}^{p} \frac{1}{n !} \int d^{d} x \int \frac{d^{d} k}{(2 \pi)^{d}} e^{-\left(\Lambda^{2}\right)^{-1} k^{2}}\left(-2\left(\Lambda^{2}\right)^{-1} T^{d} k_{d}\right)^{p}\left(2\left(\Lambda^{2}\right)^{-1} F\right)^{n-p} \\
= & -2 i \sum_{p \in \text { even }} \int d^{d} x\left[\frac{\left(\Lambda^{2}\right)^{\frac{1}{2}(d+p)}}{2^{\frac{1}{2} p}(4 \pi)^{d / 2}} \sum_{\{\sigma\}}\left(\delta_{\sigma\left(b_{1}\right) \sigma\left(b_{2}\right)} \delta_{\sigma\left(b_{3}\right) \sigma\left(b_{4}\right)} \ldots\right)\right] \frac{\left(-2 \Lambda^{-2} T^{b}\right)^{p}}{p !} e^{2\left(\Lambda^{2}\right)^{-1} F .}
\end{aligned}
$$

The $\rightarrow$ symbol in the third line is because we have replaced $-i \sigma^{a_{1} b_{1}}\left(T_{a_{1} b_{1}}^{d}+F_{a_{1} b_{1}}\right)$ with $2 T^{d}+2 F$ and $T^{d}=\frac{1}{2} T^{d}{ }_{\mu \nu} d x^{\mu} \wedge$ $d x^{\nu}, F=\frac{1}{2} F_{\mu \nu} d x^{\mu} \wedge d x^{\nu}$, which is because of the following identity

$$
\operatorname{tr}\left(\gamma^{5} \sigma^{a_{1} b_{1}} \sigma^{a_{2} b_{2}} \ldots \sigma^{a_{n} b_{n}}\right)=(-1)(-i)^{n} \epsilon^{a_{1} b_{1} \ldots a_{n} b_{n}} .
$$

In the fourth line, we perform the expansion $(a+b)^{n}=\sum_{m} C_{n}^{m} a^{m} b^{(n-m)}$. In the last line, we use the following identity

$$
\begin{aligned}
& \int \frac{d^{d} k}{(2 \pi)^{d}} \exp \left(-k_{a} \Lambda^{-2} \delta_{a b} k_{b}\right) k_{a_{1}} k_{a_{2}} \ldots k_{a_{2 m}} \\
= & \left(\frac{1}{2}\right)^{m} \frac{\left(\Lambda^{2}\right)^{d / 2+m}}{(4 \pi)^{d / 2}}\left(\sum_{\{\sigma\}} \delta_{\sigma\left(a_{1}\right) \sigma\left(a_{2}\right)} \ldots\right),
\end{aligned}
$$

where $\sum_{\{\sigma\}}$ means a sum over all possible permutations.

Notice that $T^{b_{1}}$ and $T^{b_{2}}$ in $\left(-2 \Lambda^{-2} T^{b}\right)^{p}$ commute, so the anomaly above can be recast as

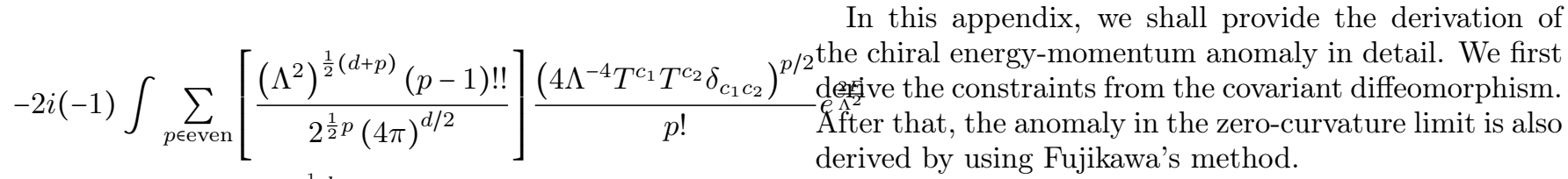
$=-2 i(-1) \int d^{d} x \frac{\left(\Lambda^{2}\right)^{\frac{1}{2} d}}{(4 \pi)^{d / 2}} e^{\Lambda^{-2} T^{c_{1}} T^{c_{2}} \delta_{c_{1} c_{2}}} e^{2 \Lambda^{-2} F}$,

where $\sum_{\sigma}\left(\delta_{\sigma\left(b_{1}\right) \sigma\left(b_{2}\right)} \delta_{\sigma\left(b_{3}\right) \sigma\left(b_{4}\right)} \ldots\right)$ contains $(p-1) ! !$ terms and $(-1)^{p}=1$ because that $p$ is an even integer. That is, we have obtained

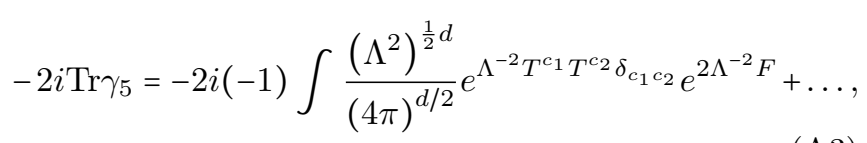

where $e^{2 \Lambda^{-2} F}$ is the Chern character and $T^{c_{1}} T^{c_{2}} \eta_{c_{1} c_{2}}$ reminds us of the Nieh-Yan term, i.e., $T^{a} \wedge T_{a}-e^{a} \wedge e^{b} \wedge \Omega_{a b}$. Thus, it is natural to conjecture that the full anomaly polynomial should be

$-2 i \operatorname{Tr} \gamma_{5}=2 i \int \frac{\left(\Lambda^{2}\right)^{\frac{1}{2} d}}{(4 \pi)^{d / 2}} e^{2 \Lambda^{-2}\left(T^{a} \wedge T^{b} \delta_{a b}-e^{a} \wedge e^{b} \wedge \Omega_{a b}\right)} e^{2 \Lambda^{-2} F} \hat{A}$

where $\hat{A}(\Omega)$ is the Dirac genus.

\section{Appendix B: Chiral energy-momentum anomaly}

\section{Covariant diffeomorphism and the corresponding current}

Consider an action $S\left(\bar{\psi}, \psi, e_{\mu}^{* a}, \omega_{a b \mu}\right)$ with both local Lorentz symmetry and local Einstein symmetry. The local Lorentz transformation is given as

$$
\begin{aligned}
& \delta e_{\mu}^{* a}=-\alpha^{a}{ }_{b} e_{\mu}^{* b}, \\
& \delta \omega_{a b \mu}=D_{\mu} \alpha_{a b},
\end{aligned}
$$

and

$$
\delta \psi=-\frac{1}{2} \alpha_{a b} \sigma^{a b} \psi
$$

where $\omega_{a b \mu}$ transforms as the gauge field for the Lorentz is

group as we expected. The local Einstein transformation

$$
\delta e_{\mu}^{* a}=\mathcal{L}_{\xi} e_{\mu}^{* a},
$$




$$
\delta \omega_{a b \mu}=\mathcal{L}_{\xi} \omega_{a b \mu},
$$

and

$$
\delta \psi=\xi^{\mu} \partial_{\mu} \psi
$$

where $\mathcal{L}_{\xi}$ is the Lie derivative of the vector $\xi=\xi^{\mu} \partial_{\mu}$. The local Einstein transformation is usually called diffeomorphism as well. By performing a local Einstein transformation and then a local Lorentz transformation with $\alpha_{a b}=\left(i_{\xi} \omega\right)_{a b}$, one can obtain the covariant diffeomorphism, i.e.,

$$
\begin{aligned}
\delta e_{\mu}^{* a} & =-T^{a}{ }_{\mu \nu} \xi^{\nu}+\nabla_{\mu} \xi^{a}, \\
\delta \omega_{a b \mu} & =-\Omega_{a b \mu \nu} \xi^{\nu} \\
\delta \psi & =\xi^{\mu} D_{\mu} \psi
\end{aligned}
$$

Then, the variation of the action

$$
\begin{aligned}
\delta S= & \int d^{d} x \sqrt{|g|}\left(-\frac{-1}{\sqrt{|g|}} \frac{\delta S}{\delta e_{\mu}^{* a}} \delta e_{\mu}^{* a}+\frac{1}{\sqrt{|g|}} \frac{\delta S}{\delta \omega_{a b \mu}} \delta \omega_{a b \mu}\right) \\
= & \int d^{d} x \sqrt{|g|}\left[\left(\nabla_{\mu}+T^{\rho}{ }_{\mu \rho}\right) \tau_{(c) a}^{\mu} \xi^{a}\right. \\
& \left.+\left(\tau_{(c) a}^{\mu} T^{a}{ }_{\mu \nu} \xi^{\nu}-S^{\mu a b} \Omega_{a b \mu} \xi^{\nu}\right)\right],
\end{aligned}
$$

where $\tau_{(c) a}^{\mu}$ is the canonical energy-momentum tensor, i.e.,

$$
\tau_{(c) a}^{\mu} \equiv \frac{-1}{\sqrt{|g|}} \frac{\delta S}{\delta e_{\mu}^{\star a}}
$$

and $S^{\mu a b}$ is the spin current, i.e.,

$$
S^{\mu a b}=\frac{1}{\sqrt{|g|}} \frac{\delta S}{\delta \omega_{a b \mu}} .
$$

Hence, we have obtained

$$
\left(\nabla_{\mu}+T_{\mu \rho}^{\rho}\right) \tau_{(c) a}^{\mu}=\left(\tau_{(c) a}^{\nu} T_{\mu \nu}^{a}-S^{\nu a b} \Omega_{a b \mu \nu}\right) e_{a}^{\mu},
$$

and similarly, for the chiral energy-momentum tensor $\tau_{(c) a}^{5 \mu}$ of the massless Dirac fermions,

$$
\left(\nabla_{\mu}+T_{\mu \rho}^{\rho}\right) \tau_{(c) a}^{5 \mu}=\left(\tau_{(c) a}^{5 \nu} T_{\mu \nu}^{a}-S^{\nu a b} \Omega_{a b \mu \nu}\right) e_{a}^{\mu} .
$$

Note that for massive Dirac fermions, the mass term would break the chiral symmetry and thus lead to an extra mass term in the equation above.

\section{Chiral energy-momentum anomalies}

After deriving the classical equation for the chiral energy-momentum tensor, we are ready to explore the corresponding quantum anomalies. For the chiral covariant diffeomorphism, i.e.,

$$
\delta \psi=\xi^{\mu} \gamma^{5} D_{\mu} \psi,
$$

one can straightforwardly obtain the Jacobian, i.e., $\mathcal{D} \psi \mathcal{D} \bar{\psi} \rightarrow J(\xi) \mathcal{D} \psi \mathcal{D} \bar{\psi}$ and

$$
\ln J(\xi)=2 \operatorname{Tr}\left(\overleftarrow{D}_{a} \gamma_{5}\right) \xi^{a}
$$

Similar to the chiral anomaly, the regularized Jacobian is

$$
\begin{aligned}
& 2 \operatorname{Tr}\left(\overleftarrow{D}_{a} \gamma^{5} e^{\Lambda^{-2} \not{D} \mathbb{D}}\right) \\
\rightarrow & -2 i(-1) \sum_{m \in Z} \frac{1}{m !} \iint \frac{d^{d} k}{(2 \pi)^{d}} e^{-t k^{2}} k_{a}\left(-2 \Lambda^{-2} T^{d} k_{d}+2 \Lambda^{-2} F\right)^{m},
\end{aligned}
$$

where $\rightarrow$ means that we have replaced $T^{d}{ }_{a b}\left(-i \sigma^{a b}\right)$ by $\frac{1}{2} T^{d}{ }_{a b} e^{a} \wedge e^{b}$ with similar notation $F$. In addition, we have kept only the most divergent term and set the spin connection to zero. Like in the chiral anomaly case, one can find that in the zero-curvature limit, the most divergent terms are

$$
2 \operatorname{Tr}\left(\overleftarrow{D}_{a} \gamma_{5}\right)=(-i) \frac{2 \Lambda^{d}}{(4 \pi)^{d / 2}} \delta_{a b} \int T^{b} e^{4 \Lambda^{-2}\left(T^{e} T^{f} \delta_{e f}\right)} e^{2 \Lambda^{-2} F}
$$

where the spacetime dimension is $2 n . T^{e} T^{f} \delta_{e f}$ in the exponent reminds us of the Nieh-Yan term, i.e., $T^{a} \wedge$ $T^{b} \eta_{a b}-e^{* a} \wedge e^{* b} \wedge \Omega_{a b}$, so in the presence of curvature, it is tempting to conjecture that

$2 \operatorname{Tr}\left(\overleftarrow{D}_{a} \gamma_{5}\right)=(-i) \frac{2 \Lambda^{2 n}}{(4 \pi)^{n}} \delta_{a b} T^{b} e^{4 \Lambda^{-2}\left(T^{a} \wedge T_{b} \delta_{a b}-e^{* a} \wedge e^{* b} \wedge \Omega_{a b}\right)} e^{2 \Lambda^{-2} F} \hat{A}(\Omega)$

where $\hat{A}(\Omega)$ is the Dirac genus.

Then, we have found that in two dimensions,

$$
\nabla_{\mu} \tau_{(c) a}^{\mu}=\frac{\Lambda^{2}}{4 \pi} \frac{\epsilon^{\mu \nu}}{\sqrt{|g|}} \eta_{a b} T_{\mu \nu}^{b}+\ldots
$$

and in $(3+1)$ dimensions,

$$
\nabla_{\mu} \tau_{(c) a}^{\mu}=\Lambda^{2} \eta_{a b} \frac{\epsilon^{\mu \nu \rho \sigma}}{\sqrt{|g|}} \frac{T_{\mu \nu}^{b}}{2 \pi} \frac{F_{\rho \sigma}}{2 \pi}+\ldots
$$

where ... means that we have kept only the most divergent terms caused by the torsion and the metric is $\eta_{a b}=\operatorname{diag}(1,-1, \ldots)$. 
[1] S. L. Adler, Phys. Rev. 177, 2426 (1969)

[2] J. S. Bell and R. Jackiw, Il Nuovo Cimento A 60, 47 (1969).

[3] S. Ryu, J. E. Moore, and A. W. W. Ludwig, Phys. Rev. B 85, 045104 (2012).

[4] E. Witten, Rev. Mod. Phys. 88, 035001 (2016)

[5] A. A. Zyuzin and A. A. Burkov, Phys. Rev. B 86, 115133 (2012).

[6] M. Stone, Quantum Hall Effect (World Scientific, 1992).

[7] K. Landsteiner, E. Megías, and F. Pena-Benitez, Phys. Rev. Lett. 107, 021601 (2011).

[8] D. T. Son and B. Z. Spivak, Phys. Rev. B 88, 104412 (2013)

[9] E. V. Gorbar, V. A. Miransky, and I. A. Shovkovy, Phys. Rev. B 89, 085126 (2014).

[10] A. Furusaki, N. Nagaosa, K. Nomura, S. Ryu, and T. Takayanagi, Comptes Rendus Physique 14, 871 (2013).

[11] Q. Li, D. E. Kharzeev, C. Zhang, Y. Huang, I. Pletikosic, A. V. Fedorov, R. D. Zhong, J. A. Schneeloch, G. D. Gu, and T. Valla, Nat. Phys. 12, 550 (2016).

[12] H. Li, H. He, H.-Z. Lu, H. Zhang, H. Liu, R. Ma, Z. Fan, S.-Q. Shen, and J. Wang, Nat. Commun. 7, 10301 (2016)

[13] Z.-M. Huang, J. Zhou, and S.-Q. Shen, Phys. Rev. B 96, $085201(2017)$

[14] J. Gooth, A. C. Niemann, T. Meng, A. G. Grushin, K. Landsteiner, B. Gotsmann, F. Menges, M. Schmidt, C. Shekhar, V. Süß, R. Hühne, B. Rellinghaus, C. Felser, B. Yan, and K. Nielsch, Nature 547, 324 (2017)

[15] M. Stone and J. Kim, Phys. Rev. D 98, 025012 (2018).

[16] M. Nakahara, Geometry, topology and physics (CRC Press, 2003).

[17] X.-L. Qi, T. L. Hughes, and S.-C. Zhang, Phys. Rev. B 78, $195424(2008)$.

[18] J. M. Luttinger, Phys. Rev. 135, A1505 (1964)

[19] G. Tatara, Phys. Rev. Lett. 114, 196601 (2015)

[20] B. Bradlyn and N. Read, Phys. Rev. B 91, 125303 (2015)

[21] M. Katanaev and I. Volovich, Annals of Physics 216, 1 (1992)

22 T. L. Hughes, R. G. Leigh, and E. Fradkin, Phys. Rev. Lett. 107, 075502 (2011)

[23] T. L. Hughes, R. G. Leigh, and O. Parrikar, Phys. Rev. D 88, 025040 (2013)

[24] O. Parrikar, T. L. Hughes, and R. G. Leigh, Phys. Rev. D 90, $105004(2014)$

[25] G. E. Volovik, The universe in a helium droplet (Oxford
University Press, 2003).

[26] O. Golan and A. Stern, Phys. Rev. B 98, 064503 (2018)

[27] J. Nissinen, arXiv preprint arXiv:1909.05846 (2019).

[28] E. V. Gorbar, V. A. Miransky, I. A. Shovkovy, and P. O. Sukhachov, Phys. Rev. B 96, 155138 (2017).

[29] Z.-M. Huang, B. Han, and M. Stone, arXiv preprint arXiv:1911.00174 (2019).

[30] H. Sumiyoshi and S. Fujimoto, Phys. Rev. Lett. 116, 166601 (2016)

[31] L. Sun and S. Wan, EPL (Europhysics Letters) 108, 37007 (2014)

[32] H. Shapourian, T. L. Hughes, and S. Ryu, Phys. Rev. B 92, 165131 (2015).

[33] Y. You, G. Y. Cho, and T. L. Hughes, Phys. Rev. B 94, $085102(2016)$

[34] G. Palumbo and J. K. Pachos, Annals of Physics 372, 175 (2016)

[35] Z.-M. Huang, L. Li, J. Zhou, and H.-H. Zhang, Phys. Rev. B 99, 155152 (2019).

[36] Z. V. Khaidukov and M. A. Zubkov, JETP Letters 108, $670(2018)$

[37] J. Nissinen and G. E. Volovik, Phys. Rev. Research 1, 023007 (2019)

[38] H. Nieh and M. Yan, Annals of Physics 138, 237 (1982).

[39] H. Nieh and M. Yan, Journal of Mathematical Physics 23, 373 (1982).

[40] Y. N. Obukhov, E. W. Mielke, J. Budczies, and F. W. Hehl, Foundations of Physics 27, 1221 (1997)

[41] O. Chandía and J. Zanelli, Phys. Rev. D 55, 7580 (1997)

[42] O. Chandía and J. Zanelli, Phys. Rev. D 58, 045014 (1998)

43 K. Peeters and A. Waldron, Journal of High Energy Physics 1999, 024 (1999)

[44] The "HLF" parity anomaly is referred to the HughesLeigh-Fradkin term in the parity-odd effective action 22 .

[45] L. Alvarez-Gaume, S. D. Pietra, and G. Moore, Annals of Physics 163, 288 (1985).

[46] J. Nissinen and G. Volovik, arXiv preprint arXiv:1911.03382 (2019).

[47] J. Nissinen and G. Volovik, arXiv preprint arXiv:1909.08936 (2019).

[48] C. G. Callan and J. A. Harvey, Nucl. Phys. B 250, 427 (1985).

[49] K. Fujikawa and H. Suzuki, Path integrals and quantum anomalies, 122 (Oxford University Press, 2004). 